\title{
Fibre Based Schemes for Ultrafast Subsystems: Nonlinear Optical Loop Mirrors Traditional Design and Novel Applications
}

\author{
Antonella Bogoni ${ }^{1}$, Francesco Fresi ${ }^{2}$, Paolo Ghelfi' ${ }^{1}$ Emma Lazzeri², \\ Luca Poti ${ }^{1}$ and Mirco Scaffardi ${ }^{1}$ \\ ${ }^{1}$ Consorzio Nazionale Interuniversitario per le Telecomunicazioni (CNIT), Pisa \\ ${ }^{2}$ Scuola Superiore Sant'Anna, Pisa \\ ${ }^{1}$ Italy \\ 2Italy
}

\section{Nonlinear Optical Loop Mirrors: an introduction}

All-optical technology is nowadays one of the most promising alternatives to electronics in various applications, ranging over a wide variety of fields such as information transmission and signal processing due to its ultrafast performances and electromagnetic interference immunity.

In this scenario, fibre-based devices are interesting because of the ultrafast response of the Kerr effects that leads to fast dynamics.

In this Chapter an exhaustive analysis of the structure of a Nonlinear Optical Loop Mirror (NOLM) is given together with the theoretical description of the phenomena behind NOLM behaviour putting emphasis on the Cross Phase Modulation (XPM) and Self Phase Modulation (SPM) effects. The theoretical description is followed by a simple and straightforward matrix model of a NOLM that allows an effortless design of multiple NOLM structures by interleaving the basic element describing the mirror. The Mickey NOLM is investigated as an example of multiple NOLM structures design and shows the potential of the model.

The Chapter also introduces novel applications by means of theoretical design and experimental results. In this section, all-optical logic gates based on NOLMs are illustrated together with the scheme of an optical decision element that allows the improvement of the receiver performances in an optical Return to Zero (RZ) transmission system and with the three stage regeneration and demultiplexing for 160-Gb/s Transmission Systems based on nonlinear optical loop mirrors.

\subsection{Structure}

Nonlinear Optical Loop Mirrors are powerful nonlinear interferometric devices often used in all-optical processing because of their ultra-fast time response due to the Kerr effect in fibre, and could therefore represent the answer to the mentioned issues. NOLMs are based 
on the Sagnac interferometer structure where the phase shift of the counterpropagating signals are unbalanced to made them interfering in a way that is suitable for the desired processing aim.

The loop is simply composed by an optical fibre coupler where the two output arms are connected to form a ring as shown in Fig. 1(a). Nonlinear elements such as Highly Nonlinear optical Fibre (HNLF) can be inserted in the loop to perform the phase shift unbalancing.
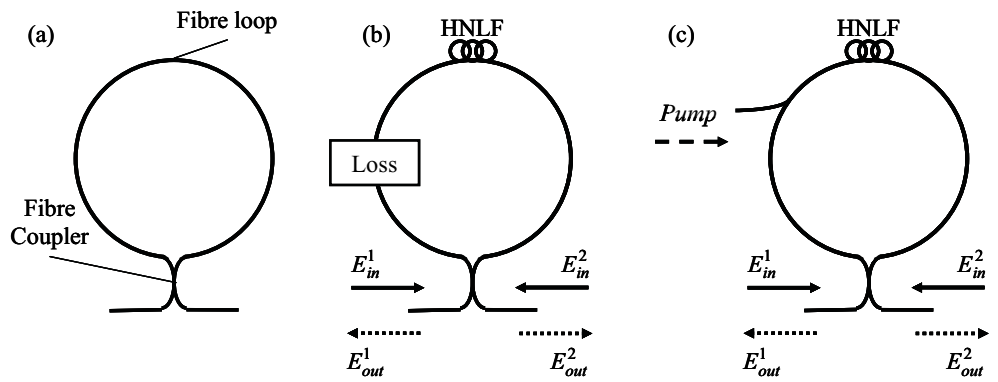

Fig. 1. (a) Main Structure of a Sagnac Interferometer; (b) HNLF-based NOLM exploiting SPM effect; (b) HNLF-based NOLM exploiting XPM effect.

\subsection{XPM and SPM}

When dealing with HNLF as nonlinear element inside the loop, two main nonlinear effects can be exploited to unbalance the phase-shifts of the counter-propagating signals interfering in the loop: Self Phase Modulation and Cross Phase Modulation.

In the former case, the fibre itself acts as nonlinear medium and a lumped loss is utilized to unbalance the two counter-propagating signal components as depicted in Fig. 1(b); since the amount of SPM (and consequently of nonlinear phase shift) experienced by a signal depends on its optical power, the presence of the lumped loss affects the system dynamic response.

In the latter case, a pump signal is introduced in the loop and circulates in only one direction as shown in Fig. 1(c); the phase shift induced in the co-propagating part of the input field depends on the instantaneous pump power, while the other portion of the input signal (counter-propagating with respect to the pump) experiences a phase shift that is determined by the pump average power.

\section{NOLM Simple and Straightforward Model}

Modular models based on basic building blocks are easy to handle when dealing with analytical description of complex physical phenomena. Assembling basic blocks allows building composite structure in a simple way and releases the model for further complications. In the model described here, each basic block is associated with a physical device and it is therefore straightforward to use.

Fig. 2(a)Left shows the structure of a Cross Phase Modulation based Polarization Maintaining NOLM (PM-NOLM) in the case of clockwise propagating pump. This interferometer was chosen to be the principal basic block of our model because of its flexibility given by the chance to use a pump signal to opportunely drive the device, 
although Self Phase Modulation based NOLM could be preferred in some application where a control signal is not required. The loop comprises a Highly Non-Linear Fibre (HNLF) of length $L_{H N L F}[\mathrm{Km}]$, non-linear coefficient $\gamma\left[\mathrm{W}^{-1} \mathrm{Km}^{-1}\right]$, negligible chromatic dispersion and dispersion slope, and two couplers, the former used to introduce the power of a pump signal at a wavelength $\lambda_{p}$ (dashed arrows) into the loop, the latter to complete the NOLM structure as well as to allow the insertion of input fields and drop of output ones. The couplers have splitting ratios $\rho_{p}$ and $\rho$ respectively.

(a)

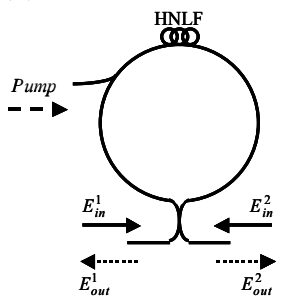

(b)

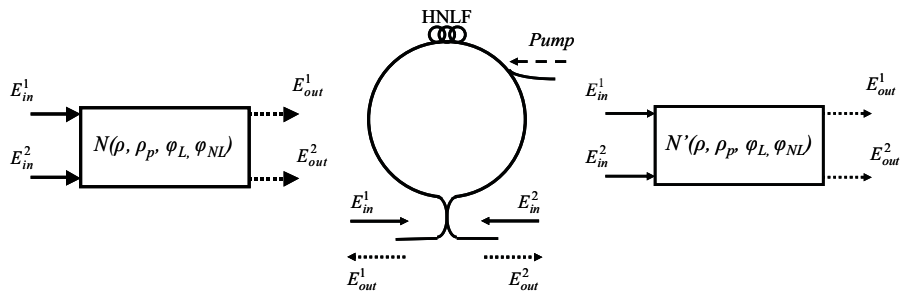

Fig. 2. (a) XPM based PM NOLM structure (Left) and its representation as a quadripole (Right) in the case of a clockwise propagating pump; (b) XPM based PM NOLM structure (Left) and its representation as a quadripole (Right) in the case of a counterclockwise propagating pump.

In a PM-NOLM all the components are polarization maintaining. Although this aspect can affect the system performances (non-PM fibres require the use of a polarization controller in the loop that adds a degree of freedom in the optimization process (Scaffardi et al., 2004), we choose to use a PM configuration in order to simplify the model that thus considers all fields as polarized along one of the fibre birefringence axes.

In our approach we describe each NOLM as a single basic quadripole defined through its dimensionless $2 \times 2$ matrix $N$. This way, each NOLM that composes the multiple structure is completely characterized and can be simply taken into account in the overall transfer function computing.

The block accepts an input vector $\overline{E_{\text {in }}}$ (solid arrows) and returns an output vector $\overline{E_{\text {out }}}$ (dotted arrows) that can be found as:

$$
\overline{E_{\text {out }}}=\left[\begin{array}{l}
E_{\text {out }}^{1} \\
E_{\text {out }}^{2}
\end{array}\right]=N \overline{E_{\text {in }}}=N\left[\begin{array}{l}
E_{\text {in }}^{1} \\
E_{\text {in }}^{2}
\end{array}\right]
$$

with:

$$
N=\left[\begin{array}{ll}
n_{1,1} & n_{1,2} \\
n_{2,1} & n_{2,2}
\end{array}\right]
$$

where:

$$
\begin{gathered}
n_{1,1}=\sqrt{\rho_{p}} e^{j \varphi_{L}} \sqrt{\rho(1-\rho)}\left(1+e^{j \varphi_{N L}}\right) e^{j \frac{\pi}{2}} \\
n_{1,2}=\sqrt{\rho_{p}} e^{j \varphi_{L}}\left(\rho-(1-\rho) e^{j \varphi_{N L}}\right) \\
n_{2,1}=\sqrt{\rho_{p}} e^{j \varphi_{L}}\left(\rho e^{j \varphi_{N L}}-(1-\rho)\right)
\end{gathered}
$$




$$
n_{2,2}=\sqrt{\rho_{p}} e^{j \varphi_{L}} \sqrt{\rho(1-\rho)}\left(1+e^{j \varphi_{N L}}\right) e^{j \frac{\pi}{2}}
$$

Equations (1)-(6) state that the PM-NOLM processes the input fields introducing two different phase shifts: a linear one, $\varphi_{L}$, which is referable to the delay caused by the loop length $L_{\text {loop }}, \varphi_{L}=\beta L_{\text {loop }}$ (where $\beta$ is the propagation constant of the field into the optical fibre) and a nonlinear one, $\varphi_{N L}$, that is due to the XPM effect induced by the pump power in the highly non linear fibre as stated in the following equation:

$$
\varphi_{N L}=2 \gamma\left(1-\rho_{p}\right) L_{H N L F} P_{p}
$$

where $P_{p}$ is the instantaneous pump power and the coupler loss has been taken into account All the elements $n_{i j}\left(\varphi_{N L}\right), i, j=1,2$ are periodic functions of period $2 \Pi$.

The model assumes the input signals to be continuous waves at a certain wavelength $\lambda_{\text {in }} \neq \lambda_{p}$ and, for the sake of simplicity, it neglects nonlinearities due to counter-propagating signals. This last simplification can be done considering the pump average power low enough, as in the case of a pulsed train with an appropriate duty cycle (Bogoni et al., 2004 a). Due to the low value for D and S, walk-off effect is also neglected.

The model validity domain can be estimated by considering following standard fibre parameters: $L_{H N L F}=0.5 \mathrm{Km}, \gamma=10 \mathrm{~W}^{-1} \mathrm{Km}^{-1}, \mathrm{D}<0.1 \mathrm{ps} \cdot \mathrm{nm}^{-1} \mathrm{~km}^{-1}$, and $\mathrm{S}<0.01 \mathrm{ps}^{2} \cdot \mathrm{nm}^{-1} \mathrm{~km}^{-1}$. Accumulated chromatic dispersion $\mathrm{D}_{\text {tot }}<0.05 \mathrm{ps} \cdot \mathrm{nm}^{-1}$ gives $175 \mathrm{fs}$ pulse broadening for a 1 ps, $3.5 \mathrm{~nm}$ Gaussian pulse width or, similarly, a walk-off of about 1 ps when spectral displacement between interacting signal is $20 \mathrm{~nm}$.

(a)

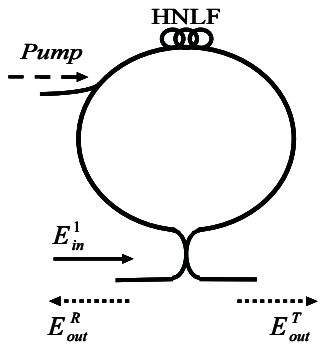

(c)

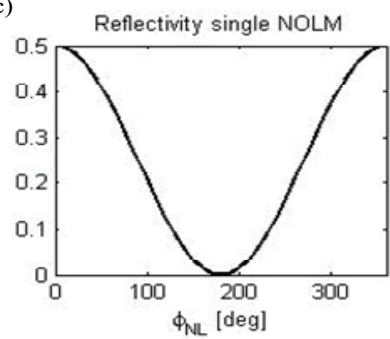

(b)

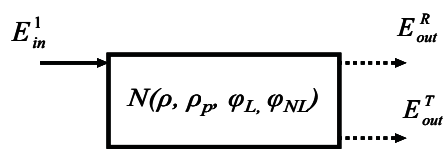

(d)

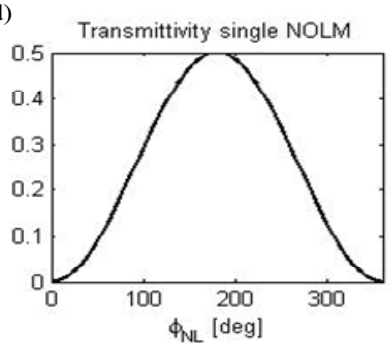

Fig. 3. XPM based PM NOLM structure (a), its representation as a quadripole (b). Reflectivity (c) and Transmittivity (d) functions in the case of a clockwise propagating pump with a single input Field. 
On the other hand, phase shift due to SPM effect is limited to $Y L_{H N L F} P_{\text {in }}$ and can be considered negligible if lower than $10^{\circ}$ thus giving an upper bound for input signal power of about $15 \mathrm{dBm}$.

Fig. 2(b) shows a XPM based PM NOLM structure and its representation as a quadripole in the case of counter-clockwise propagating pump. Assuming the same references for the input and output fields as in Fig. 2(a), it is possible to demonstrate that the dimensionless matrix that describes the NOLM corresponds to the transpose of $N$, i.e. $N^{\prime}$.

The model introduced above can be used to describe a single NOLM as Fig. 3 shows. The results suppose the structure to be fed with a single input field $\left(E^{2}{ }_{i n}=0\right)$ represented by a continuous wave at $\lambda_{i n}=1550 \mathrm{~nm}$. Fig. 3 shows both the Reflectivity and the Transmittivity as functions of the nonlinear phase shift induced by the pump power as stated in (7).

\section{Multiple Structure Design}
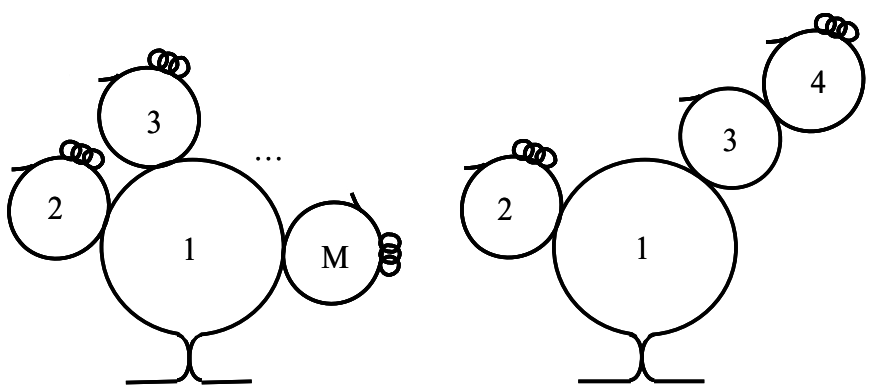

Fig. 4. Example of multiple NOLM device schemes. Left - A principal NOLM structure is connected to M-1 single NOLMs. Right - NOLM no. 1 is united to two separated NOLMs (no. 2 and no.3), while NOLM no. 4 is attached only to NOLM no.3.

Once the modular model is verified for single and cascaded NOLMs, more complex structures can be considered in order to investigate for new schemes where a simple parameters tuning can lead to considerable changes in the output functions. Single NOLM response in terms of Transmittivity and Reflectivity have a sinusoidal shape, whilst numerical (Nayar et al., 1991) and experimental (Nayar et al., 1993) investigations have shown that cascading several NOLMs leads to a sharpening of the overall switching characteristics that results in steeper switching edges and flatter peaks. Single or cascaded NOLMs structures are therefore used to perform diverse all-optical processing tasks such as wavelength conversion (Sakamoto et al., 2001) and to build important network devices like soliton switches (Pattison et al., 1995). However, to the best of our knowledge, no work has been done to describe analytically or experimentally the performance of multiple and interleaved NOLM configurations such as the ones shown in Fig. 4.

\subsection{Interleaving the basic element}

Multiple and interleaved NOLM configurations can be composed by interconnecting the basic block defined in this Section with other elements such as fibre spans and couplers; for this purpose these elements can be described with matrices as reported in equations (8) and 
(9), where $C$ models the behaviour of a coupler with splitting ratio $\rho$ and $D$ represents the phase shift induced by a fibre span of length $L_{F}$.

$$
\begin{gathered}
C=\left[\begin{array}{cc}
\sqrt{\rho} & \sqrt{1-\rho} e^{j \frac{\pi}{2}} \\
\sqrt{1-\rho} e^{j \frac{\pi}{2}} & \sqrt{\rho}
\end{array}\right] \\
D=\left[e^{j \theta}\right], \theta=\beta L_{F} .
\end{gathered}
$$

The corresponding blocks are depicted in Fig. 5 that shows the structure, block diagram and results of a two XPM based PM NOLMs cascade fed with a single input field $\left(E^{2}{ }_{i n}=0\right)$ represented by a continuous wave at $\lambda_{i n}=1550 \mathrm{~nm}$. In this case, Transmittivity and Reflectivity were studied as functions of the normalized pump powers defined as:

$$
P_{p}^{k} \Gamma=\varphi_{N L}^{k}
$$

where:

$$
\Gamma=2 \gamma\left(1-\rho_{p}^{k}\right)
$$

By introducing a linear dependence between the normalized pump powers, different Reflectivity and Transmittivity curves can be extracted from the bi-dimensional plots as shown in Fig. 6(c). In order to give an accurate performance comparison with the corresponding single NOLM characteristics the curves are plotted as functions of the normalized total pump power:

$$
\text { Ptot } \Gamma=\left(P_{p}^{1}+P_{p}^{2}\right) \Gamma=P_{p}^{2}(m+1) \Gamma=\varphi_{N L}^{2}(m+1)
$$

The linear dependence $P_{p}{ }^{2}=P_{p}{ }^{1} m$ is shown by the black superimposed line on the bidimensional plots. All curves are compared with the corresponding single NOLM $T$ or $R$ functions (dashed curves) that have sinusoidal shapes. Simulations were run using following parameters: $\varphi^{1}{ }_{L}=0^{\circ}, \varphi^{2}{ }_{L}=0^{\circ}, \theta=0^{\circ}, m=1$.

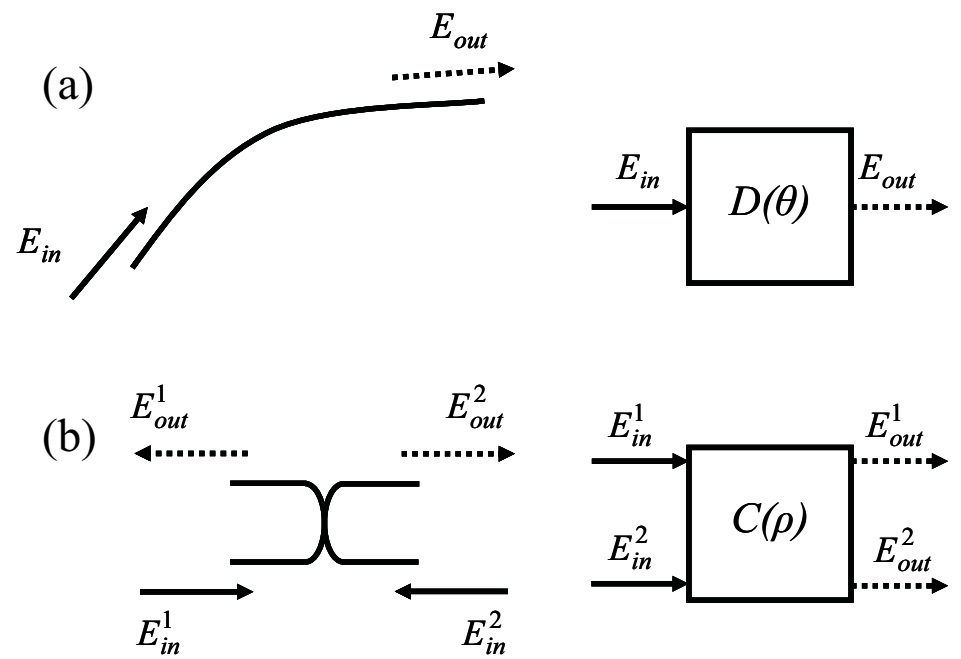

Fig. 5. (a) - Fibre span (left) and block representation (right); (b) - Coupler (left) and block representation (right). 
(a)

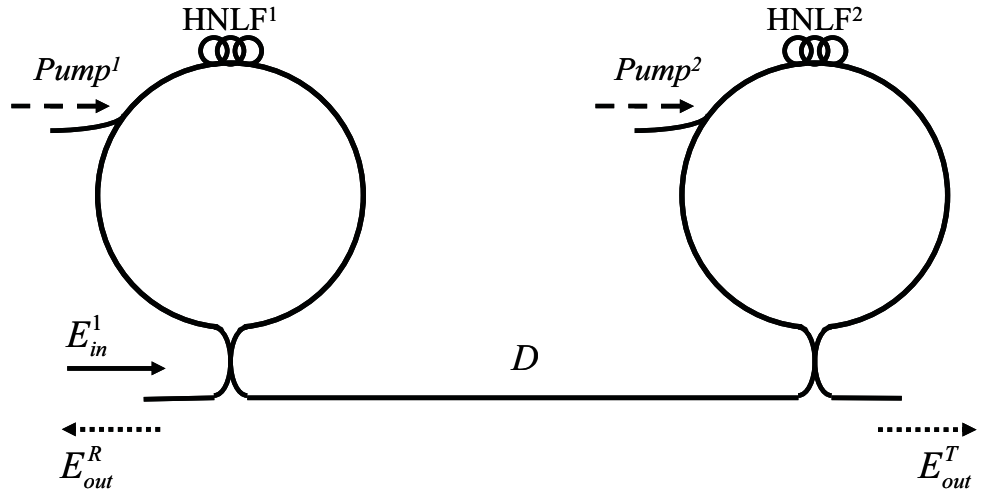

(b)

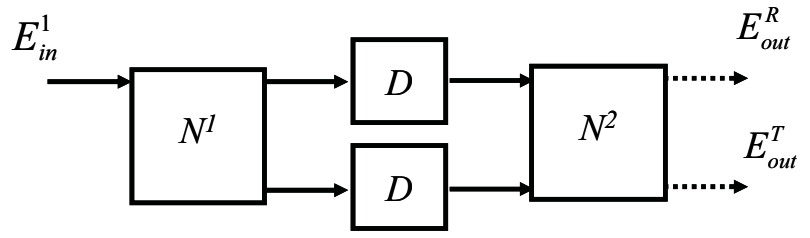

(c)
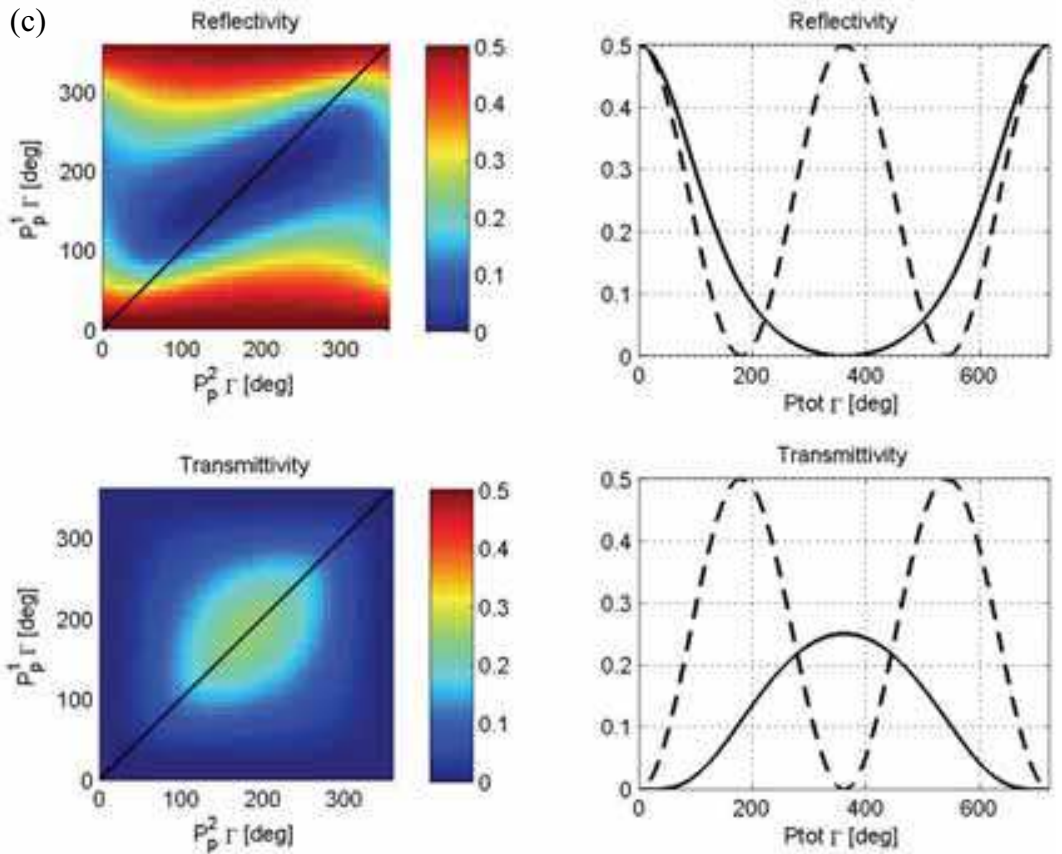

Fig. 6. Cascade of two XPM based PM NOLMs structure (a), block diagram (b) and simulation results (c) in the case of a clockwise propagating pumps with a single input Field. 


\subsection{Mickey NOLM}

A particular configuration, which we will address to as Mickey NOLM, was designed to test the model described in section II. The structure of Mickey NOLM is shown in Fig. 7. Considering the NOLM versions related to clockwise and counter-clockwise propagating pumps, we can imagine to have four diverse configurations corresponding to the possible XPM-based PM NOLMs combinations. Ignoring the redundant configurations due to the possible input and output ports switch, we end up with only three versions of the Mickey NOLM that are shown in Fig. 7.

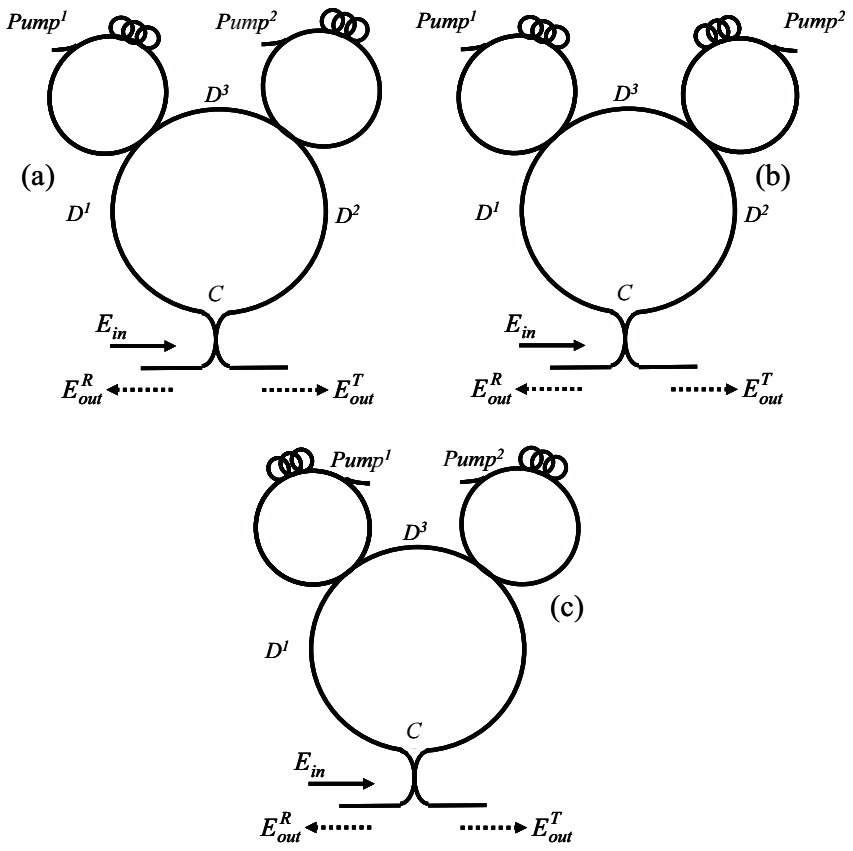

Fig. 7. Mickey NOLM possible structures; the three versions differ from each other in the pumps propagating directions; (a) - the XPM-based PM NOLMs both present clockwise propagating pumps; (b) - the XPM-based PM NOLM on the left side of the device present clockwise propagating pump while the one on the right makes use of counter-clockwise propagating pump; (c) - the XPM-based PM NOLM on the right side of the device present clockwise propagating pump while the one on the left makes use of counter-clockwise propagating pump.

To compose the Mickey NOLM, two XPM-based PM NOLM are connected with three fibre spans of certain lengths $L_{F}^{m}(m=1,2,3)$ and one coupler $(C(\rho)$ as in equation (8)). One input field is fed into the structure (solid arrow in Fig. 7) to produce two outputs: the transmitted and the reflected fields (dotted arrows in Fig. 7). Our interest is focused on Reflectivity (R) and Transmittivity (T) as functions of the nonlinear phase shifts $\varphi_{N L}^{1}, \varphi_{N L}^{2}$ that can be easily 
ascribed to the pump powers $\left(P_{p}^{1}, P_{p}^{2}\right)$ by linear conversion as established in equation (7)); to compute $\mathrm{R}$ and $\mathrm{T}$, we first need to outline the Mickey NOLM block diagram by means of the basic elements shown in Figures Fig. 2Fig. 5. The resulting diagram is shown in Fig. 8(a) where $\mathrm{N} \mathrm{k}\left(\mathrm{k}=1\right.$, 2.) indicate the XPM-based PM NOLMs, $D^{m}=\left\lfloor e^{j \theta^{m}}\right\rfloor, \theta^{m}=\beta L_{F}^{m}$ indicate the three different fibre spans, and $\mathrm{C}$ is the coupler as described in (8).

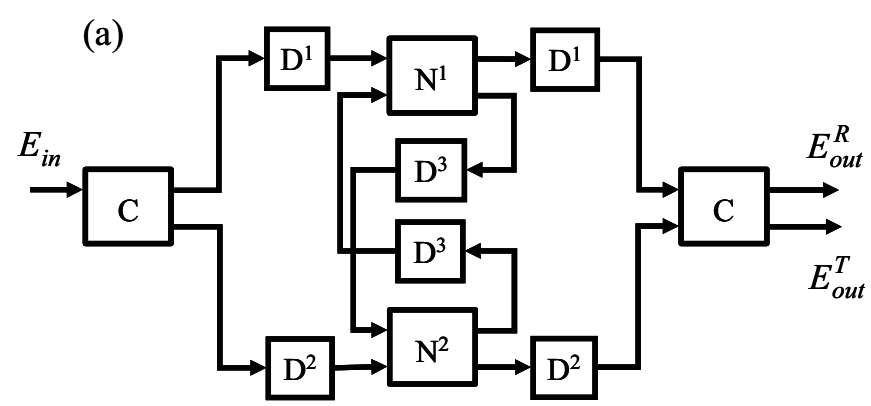

(b)

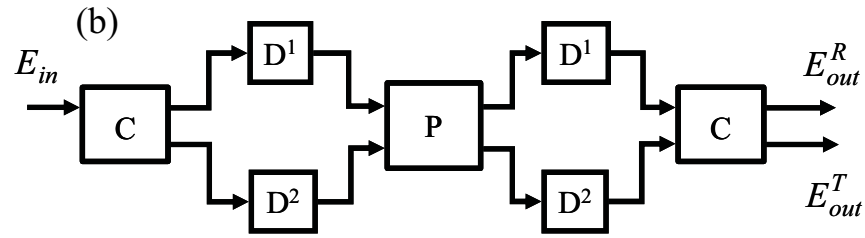

(c)

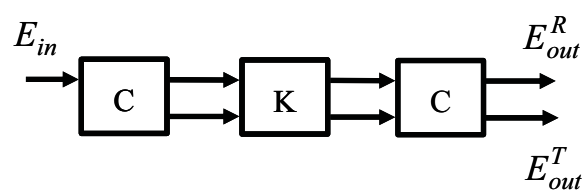

Fig. 8. Mickey NOLM block diagram.

Linear phase shifts introduced in the structure depend on the particular fibre span or loop lengths, and are considered as parameters in the following equations that define the Transmittivity $(T)$ and the Reflectivity $(R)$ :

$$
\begin{aligned}
& T\left(\varphi_{N L}^{1}, \varphi_{N L}^{2} ; \varphi_{L}^{1}, \varphi_{L}^{2}, \theta^{1}, \theta^{2}, \theta^{3}\right)=\frac{P_{\text {out }}^{T}}{P_{\text {in }}}=\frac{\left|E_{\text {out }}^{T}\right|^{2}}{\left|E_{\text {in }}\right|^{2}} ; \\
& R\left(\varphi_{N L}^{1}, \varphi_{N L}^{2} ; \varphi_{L}^{1}, \varphi_{L}^{2}, \theta^{1}, \theta^{2}, \theta^{3}\right)=\frac{P_{\text {out }}^{R}}{P_{\text {in }}}=\frac{\left|E_{\text {out }}^{R}\right|^{2}}{\left|E_{\text {in }}\right|^{2}} .
\end{aligned}
$$


To find the complete $R$ and $T$ expressions, the block diagram in Fig. 8(a) can be simplified as shown in Fig. 8(b), where block P substitutes $N^{1}, N^{2}$ and $D^{3}$, and is defined by the following 2x2 matrix:

$$
P=\left[\begin{array}{ll}
p_{1,1} & p_{1,2} \\
p_{2,1} & p_{2,2}
\end{array}\right]
$$

where:

$$
\begin{gathered}
p_{1,1}=\left(n_{1,1}^{1}+\frac{n_{1,2}^{1} n_{1,1}^{2} n_{2,1}^{1} e^{j 2 \theta^{3}}}{1-n_{1,1}^{2} n_{2,2}^{1} e^{j 2 \theta^{3}}}\right) \\
p_{1,2}=\left(\frac{n_{1,2}^{1} n_{1,2}^{2} e^{j \theta^{3}}}{1-n_{1,1}^{2} n_{2,2}^{1} e^{j 2 \theta^{3}}}\right) \\
p_{2,1}=\left(\frac{n_{2,1}^{1} n_{2,1}^{2} e^{j \theta^{3}}}{1-n_{1,1}^{2} n_{2,2}^{1} e^{j 2 \theta^{3}}}\right) \\
p_{2,2}=\left(n_{2,2}^{2}+\frac{n_{2,1}^{2} n_{2,2}^{1} n_{1,2}^{2} e^{j 2 \theta^{3}}}{1-n_{1,1}^{2} n_{2,2}^{1} e^{j 2 \theta^{3}}}\right)
\end{gathered}
$$

Furthermore, block $\mathrm{K}$ in Fig. 8(c) replaces $\mathrm{P}, \mathrm{D}^{1}$ and $\mathrm{D}^{2}$ blocks, and it is characterized by the matrix defined in ():

$$
K=\left[\begin{array}{ll}
k_{1,1} & k_{1,2} \\
k_{2,1} & k_{2,2}
\end{array}\right]
$$

where:

$$
\begin{gathered}
k_{1,1}=e^{j 2 \theta^{1}} p_{1,1} \\
k_{1,2}=e^{j\left(\theta^{1}+\theta^{2}\right)} p_{1,2} \\
k_{2,1}=e^{j\left(\theta^{1}+\theta^{2}\right)} p_{2,1} \\
k_{2,2}=e^{j 2 \theta^{2}} p_{2,2}
\end{gathered}
$$

Finally, the expressions of $T$ and $R$ can be obtained by matrix computing:

$$
\overline{E_{\text {out }}}=\left[\begin{array}{c}
E_{\text {out }}^{R} \\
E_{\text {out }}^{T}
\end{array}\right]=C K C\left[\begin{array}{c}
E_{\text {in }} \\
0
\end{array}\right]
$$

The null value in the input vector, states that only one input field has been considered.

By substituting expressions (1)-(6), (8)-(9), (14)-(24) in (13) we find that the Transmittivity and Reflectivity functions depend on the phase difference $\Delta \theta=\theta^{1}-\theta^{2}$ and not on the single linear phase shifts, thus resulting in a dependence of $T$ and $R$ on the relative length of paths $D^{1}$ and $D^{2}$ and not on the specific lengths of the fibre spans:

$$
\begin{aligned}
& T=T\left(\varphi_{N L}^{1}, \varphi_{N L}^{2} ; \varphi_{L}^{1}, \varphi_{L}^{2}, \Delta \theta, \theta^{3}\right) ; \\
& R=R\left(\varphi_{N L}^{1}, \varphi_{N L}^{2} ; \varphi_{L}^{1}, \varphi_{L}^{2}, \Delta \theta, \theta^{3}\right) .
\end{aligned}
$$

As a result of $n_{i j}^{k_{i j}}\left(\varphi^{k}{ }_{N L}\right), i, j, k=1,2$ periodicity, $T$ and $R$ are periodic functions of $\varphi^{1} N L$ and $\varphi^{2} N L$ of period 2п. 
By a proper linear phase shifts tuning, different Transmittivity and Reflectivity functions can be obtained. To opportunely tune the linear phase shifts, optical delay lines can be used. In order to reduce the number of tunable parameters in our simulations, we fixed all the couplers splitting ratios equal to 0.5 and we considered the HNLF to be of equal length $L_{H N L F}$ and to have the same nonlinear coefficient $\gamma$. We show here a set of results obtained for different configurations and parameters values.
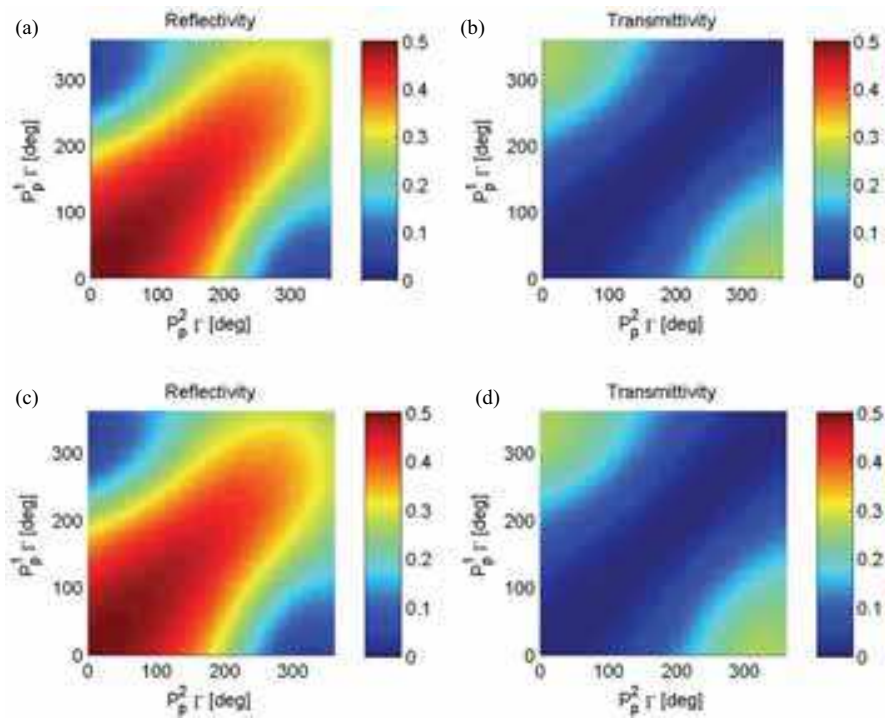

Fig. 9. All figures are obtained with: $\varphi_{L}{ }^{1}=0^{\circ}, \varphi_{L^{2}}=20^{\circ}, \theta^{3}=10^{\circ}, \Delta \theta=280^{\circ}$ applied to Fig. 7 (a) structure; (a) - Reflectivity for $\theta^{1}=280^{\circ}$ and $\theta^{2}=0^{\circ}$; (b) - Transmittivity for $\theta^{1}=280^{\circ}$ and $\theta^{2}=0^{\circ}$; (c) - Reflectivity for $\theta^{1}=0^{\circ}$ and $\theta^{2}=80^{\circ}$; (d) -Transmittivity for $\theta^{1}=0^{\circ}$ and $\theta^{2}=80^{\circ}$. Mickey NOLM block diagram.

All results suppose the input field $E_{\text {in }}$ to be a continuous wave at $\lambda_{i n}=1550 \mathrm{~nm}$.

The property stated in equation (25) can be confirmed graphically as in Fig. 9, where the transfer functions ( $R$ and $T$ ) are shown for two different sets of linear phase shifts: while $\varphi_{L}{ }^{1}$, $\varphi_{L}{ }^{2}, \theta^{3}$ and $\Delta \theta$ are kept constant, the values of $\theta^{1}$ and $\theta^{2}$ are varied, but the overall Transmittivity and Reflectivity functions do not differ.

Fig. 10-Fig. 12 show the Transmittivity and Reflectivity bi-dimensional plots and the corresponding curves obtained by introducing a linear dependence between the pump powers.

A scheme with a steep soft limiting function, like the one reported in Fig. 10, can be used as an efficient in-line data regenerator of the pump signals. Fig. 10 shows a $T$-curve that can be exploited as logical port: the port input signals $S_{1}(t)$ and $S_{2}(t)$ are combined to form the pump signals that are exactly the same for the two XPM-based NOLM blocks. Fig. 11(c) shows the truth table of the corresponding NOR logic gate. In this case the single port can be physically realized by means of a $3 \mathrm{~dB}$ coupler that adds $S_{1}(t)$ and $S_{2}(t)$ and then splits the signal obtained in order to produce two identical pump signals. 
Steeper curves can be obtained by tuning the set of parameters as in Fig. 12; this aspect is quite important because steeper curves mean that lower power is needed to cover the whole switching characteristic
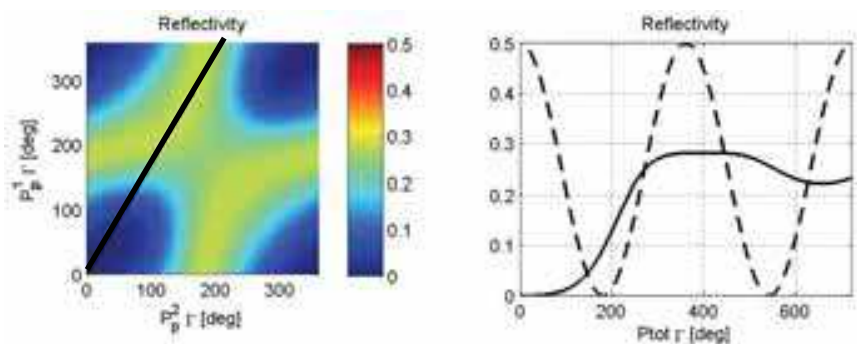

Fig. 10. Parameters used in the simulation: $\varphi^{1}{ }_{L}=0^{\circ}, \varphi^{2}{ }_{L}=20^{\circ}, \theta^{3}=170^{\circ}, \Delta \theta=10^{\circ}, m=1.71$ applied to Fig. 7(a) structure; results are compared to the Reflectivity of a single XPM-based NOLM (dashed curve); Left - Reflectivity bi-dimensional plot as function of normalized pump powers; Right - R-curve (solid curve) obtained by introducing a linear dependence between the pump powers that can be exploited in in-line pulse train regeneration .

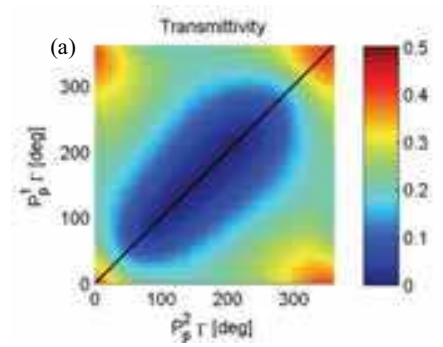

(b)

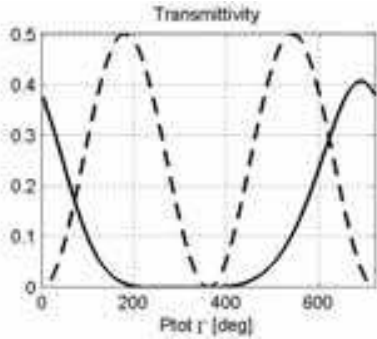

(c) Ptot $\Gamma=S_{1}(t)+S_{2}(t)$

\begin{tabular}{|c|c|c|}
\hline $\mathrm{S}_{1}(\mathrm{t})$ & $\mathrm{S}_{2}(\mathrm{t})$ & $\begin{array}{c}\text { Out } \\
\text { (NOR) }\end{array}$ \\
\hline 0 & 0 & 1 \\
\hline 0 & 1 & 0 \\
\hline 1 & 0 & 0 \\
\hline 1 & 1 & 0 \\
\hline
\end{tabular}

Fig. 11. Parameters used in the simulation: $\varphi^{1}{ }_{L}=0^{\circ}, \varphi^{2}{ }^{2}=20^{\circ}, \theta^{3}=170^{\circ}, \Delta \theta=70^{\circ}, m=1.00$ applied to Fig. 7(c) structure; results are compared to the Transmittivity of a single XPM-based NOLM (dashed curve); (a) - Transmittivity bi-dimensional plot as function of normalized pump powers; (a) - T-curve obtained by introducing a linear dependence between the pump powers that can be exploited for building a NOR logic gate as truth table explains (c).

Fig. 13 shows a different use of the bi-dimensional plot: here the pump powers are utilized as binary input signal for a NOR logical gate. 

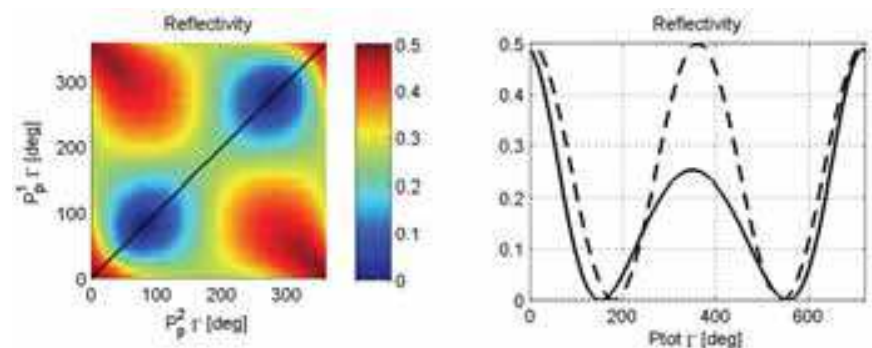

Fig. 12. Parameters used in the simulation: $\varphi^{1}{ }_{L}=0^{\circ}, \varphi^{2}{ }_{L}=20^{\circ}, \theta^{3}=170^{\circ}, \Delta \theta=0^{\circ}, m=0.99$; results are compared to the Reflectivity of a single XPM-based NOLM (dashed curve); Left Reflectivity bi-dimensional plot as function of normalized pump powers; Right $-R$-curve obtained by introducing a linear dependence between the pump powers that shows fast dynamics

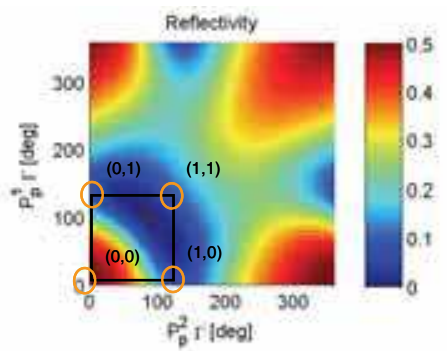

\begin{tabular}{|c|c|c|}
\hline $\mathrm{P}_{\mathrm{p}}^{2}$ & $\mathrm{P}_{\mathrm{p}}^{\mathrm{l}}$ & Out (NOR) \\
\hline 0 & 0 & 1 \\
\hline 0 & 1 & 0 \\
\hline 1 & 0 & 0 \\
\hline 1 & 1 & 0 \\
\hline
\end{tabular}

Fig. 13. Parameters used in the simulation: $\varphi^{1}{ }_{L}=0^{\circ}, \varphi^{2}{ }_{L}=190^{\circ}, \theta^{3}=130^{\circ}, \Delta \theta=0^{\circ}$ applied to Fig. 7(c) structure; Reflectivity bi-dimensional plot as function of normalized pump powers (Left) exploited for building a NOR logic gate as truth table explains (Right); in this case the pump signals are used as input signal of the logical port.

\section{Novel Experimental Applications}

\subsection{Logic Gates}

All-optical digital logic gates are indispensable in the ultra-fast all-optical signal processing. In literature examples of all-optical logic gates have been presented, exploiting nonlinear effects in semiconductor devices (Stubkjaer 2000; Ibrahim et al., 2003; Webb et al., 2003; Hall \& Rauschenbach, 1998), optical fibre (Olsson \& Andrekson, 1998; Ahn et al., 1997; Chbat et al., 1992), or waveguides (Collecutt \& Drummond, 2000) .Self Phase Modulation and Cross Phase Modulation in Dispersion Shifted Fibre (DSF) can be used in Nonlinear Optical Loop Mirror to obtain AND/OR/XOR, and NOR/XNOR functions respectively. These same structures have been presented in (Olsson \& Andrekson, 1998; Ahn et al., 1997) to obtain reconfigurable logic gates with advantages in term of flexibility and low complexity.

With reference to the NOLM behaviour (Bogoni et al., 2004 a; Agrawal, 1994), if the NOLM is built with non-Polarization-Maintaining (non-PM) fibre, a polarization controller into the loop can change the NOLM characteristic (power of the signal inducing the phase shift in DSF versus the output power), adding a constant shift of the phase difference between the counterpropagating signals at the output of the loop. This shift allows changing the pump 
power levels that produce constructive or destructive interference at the output. This property due to the interaction between the linear transformation operated by the polarization controller and the nonlinear effect in the DSF (Bogoni et al., 2004 a), can be exploited in order to obtain different logic functions with the same scheme.
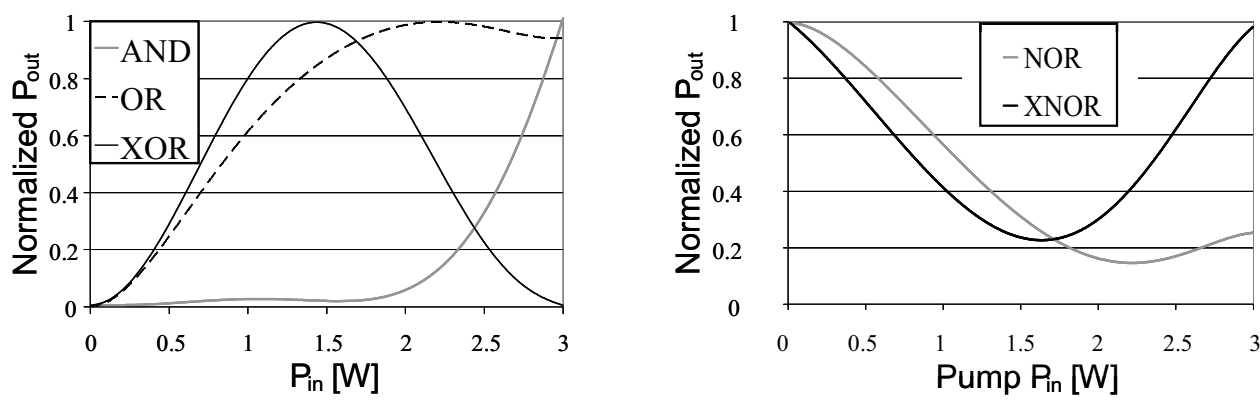

Fig. 14. Nonlinear characteristics of a SPM-based (top) and XPM-based (bottom) NOLM scheme.

Fig. 14 shows the different nonlinear characteristics that can be obtained with the SPMbased (left) and a XPM-based (right) NOLM reported in Fig. 15, by opportunely adjusting the polarization controller into the loop. If we consider the signal inducing the phase shift in the DSF, (abscissa in the plot) as the sum of two digital signals A and B, both with the low level equal to $0 \mathrm{~W}$ and the high level at $1.5 \mathrm{~W}$, the characteristics in the figure represent the logic function AND, OR, XOR (left) and XNOR, NOR (right) of the signals A and B. Note that the non-PM fibre implementation in the case of XPM-based NOLM allows the realization of inverting functions (Bogoni et al., 2004 a). The optimization of the polarization controller state can be experimentally made. In Fig. 14 it is also possible to evaluate the onoff contrast ratio for each considered logic gate: in the best cases (AND, XOR, OR) the ratio is more than $20 \mathrm{~dB}$, while in the worst case (XNOR) it is higher than $6.5 \mathrm{~dB}$ due to the pedestal of the output signal. Anyway, the contrast ratio can be further improved by adding a SPM-based NOLM in cascade, acting as pedestal suppressor (Bogoni et al., 2004 b).
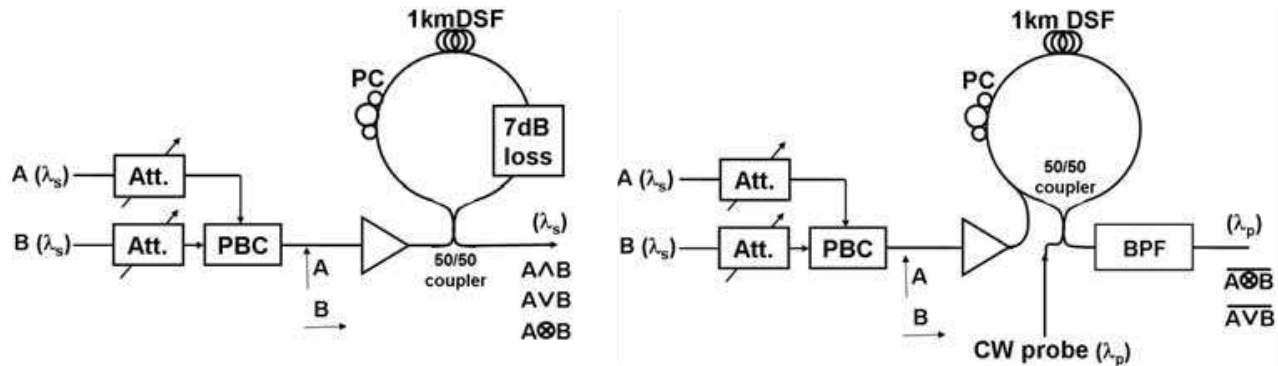

Fig. 15. Experimental set-up for AND/OR/XOR (left) and XNOR/NOR (right) gates.

Fig. 15 shows the experimental set-up of the all-optical logic gates. The signals A and B are combined at the same wavelength, in order to avoid four wave mixing in the DSF and walk- 
off impairments, and with orthogonal polarization in order to eliminate instability due to phase interference.
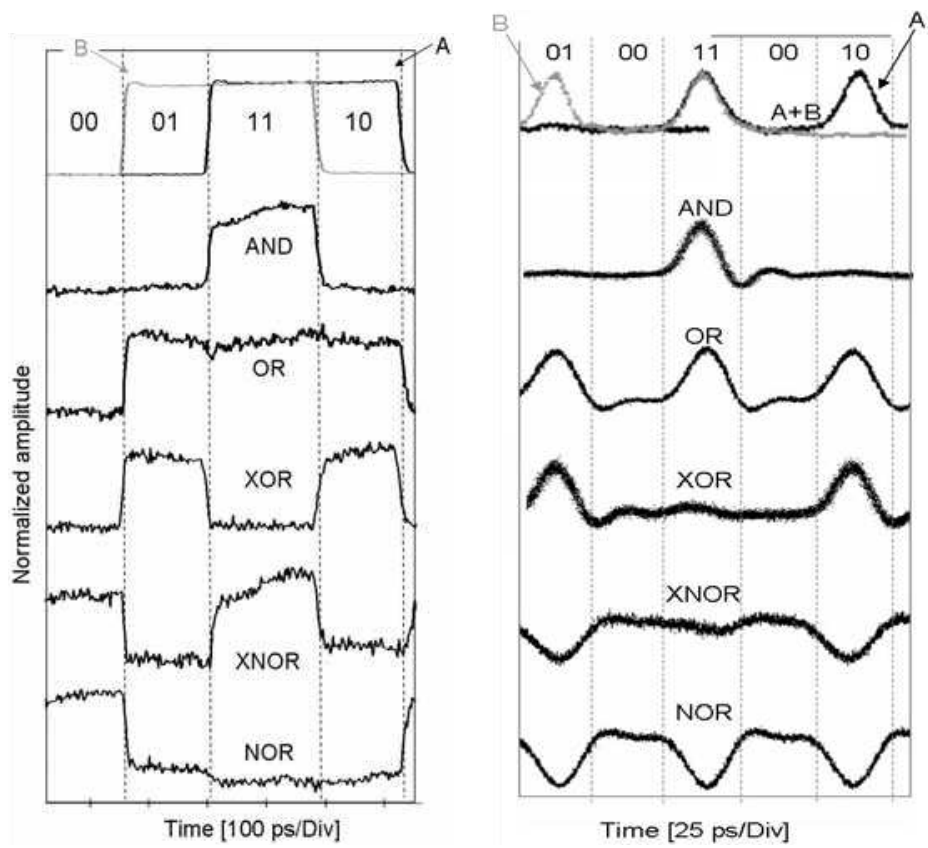

Fig. 16. Signal A and B at the input of the all-optical logic gates (top) and corresponding output of AND, OR, XOR, XNOR, and NOR (bottom) for NRZ (left) and RZ (right) signals; RZ Results obtained by means of $4 \mathrm{ps}$ wide pulses.

Fig. 16 shows the normalized time behaviour of the signals $\mathrm{A}$ and $\mathrm{B}$ at the input of the implemented all-optical logic gates (top) and the normalized outputs (bottom), considering $10 \mathrm{GHz}$ Non Return to Zero (NRZ) (left) and $40 \mathrm{GHz}$ RZ (right) signals. In a NOLM the counter-propagating light experiences SPM/XPM due to nonlinear interaction between counter-propagating signals, depending on the mean power of the light responsible for the effect (Chbat et al., 1992). If the duty-cycle $d$ of the signal inducing SPM or XPM is low (lowbit rate ultra-short pulsed signal), this effect can be neglected, since the mean power is much smaller than the peak power. But when the duty-cycle gets higher these effects rapidly increase and they can strongly affect the NOLM performance. The use of a NRZ modulation format (high $d$ value $\approx 0.5$ ) allows verifying the effectiveness of a non-PM loop implementation, compensating for undesirable counter-propagating effects (Chbat et al., 1992). The AND, XOR, and OR logic gates present also regenerative properties. In fact an improvement of $3 \mathrm{~dB}$ of the output signal on-off contrast ratio has been measured with respect to the $17 \mathrm{~dB}$ on-off contrast ratio of the input signals. This improvement is due to the capability of the SPM-based NOLM to act as pedestal suppressor (Bogoni et al., $2004 \mathrm{~b}$ ). 
Note that here the input signals have been intentionally generated with a not negligible zero level in order to obtain a on-off contrast ratio of $17 \mathrm{~dB}$.

Finally, considering an input Q-factor of 5 for both A and B signals, an increase in the output Q-factor between 1 and 2 has been measured for all the logic gates but the XNOR, which, on the other hand, has shown a decrease of 1 . This Q-factor decrease due to the pedestal of the output signal can be avoided inserting a pedestal suppressor stage. Note that the Q-factor in this case directly gives the scheme performances without strong relationship with any system bit error rate (BER).

\subsection{Optical Decision Element}

The development of new generation optical networks is gathering increasing interest towards Return to Zero data formats, since they will be used in both systems exploiting Optical Time Division Multiplexing (OTDM) and Wavelength Division Multiplexing (WDM) (Rhode et al., 2004; Murakami et al., 1998). Optical RZ signals present a bandwidth $B$ exceeding the bit rate $R$ of the single channel, and the bandwidth of the optimum RZ receiver should be proportional to $B$ (Winzer et al., 2001), rather than to $R$ as for NRZ data formats. Therefore, since future increases in RZ system capacity would require pulsed signals with increasing bandwidths, the optimum conventional RZ receivers will consequently need expensive very fast electronic and optoelectronic components to match the input signal characteristics, even in the case of relatively low bit rates as for demultiplexed OTDM channels. In this scenario an attractive alternative to conventional optimum receivers, which performs an electrical decision, could be the use of an additional all-optical ultra-fast decision element just before the conventional band-limited receiver, as shown in Fig. 17. This way, a first decision is carried out in the optical domain without any bandwidth limitation, improving the quality of the signal to be received. The very large bandwidth of all-optical nonlinear phenomena, together with the possibility to develop bit rate transparent, integrable and low-cost optical schemes, make this solution competitive with the conventional optimum receiver.

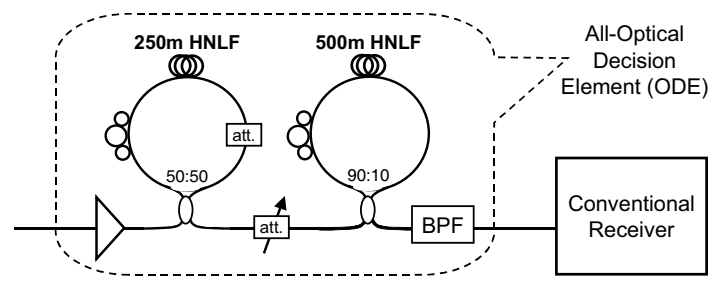

Fig. 17. Scheme of the proposed receiver. The HNLF has a nonlinear coefficient $\gamma=10 \mathrm{~W}^{-1} \mathrm{~km}^{-1}$ and $\lambda_{0}=1553 \mathrm{~nm}$. Attenuation in the first NOLM: $2.5 \mathrm{~dB}$.

A decision element can be obtained in the optical domain exploiting the nonlinear characteristic of NOLM structures (Bogoni et al., 2004 b), which allow faster operations compared to other schemes, based for example on semiconductor devices. Moreover the possibility to use short spans of new highly nonlinear fibres allows increasing the stability and efficiency of the interferometric scheme. Fig. 17 shows the implemented ODE, constituted by an Erbium Doped Fibre Amplifier (EDFA) followed by two cascaded 
NOLMs. The first one exploits a $3 \mathrm{~dB}$ coupler and 250m of Highly Non-Linear Fibre (Bogoni et al., $2004 \mathrm{~b}$ ). The second NOLM comprises a 10/90 coupler and 500m of HNLF (Meissner et al., 2003). Finally an optical filter eliminates the out-of-band Amplified Spontaneous Emission (ASE) noise. Note that this configuration allows using just one EDFA at the input of the first NOLM, since the power required by the second fibre loop is available at the output of the first NOLM without further amplification.
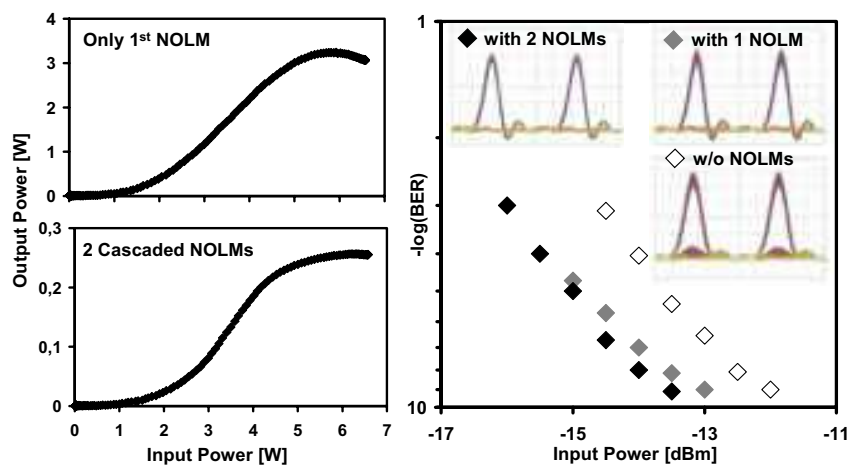

Fig. 18. Left: nonlinear characteristic of the ODE made with 1 and 2 NOLMs. Right: BER measured for $0.3 \mathrm{~nm}$ bandwidth input signal, with OSNR=15dB. Insets: eye-diagrams at the receiver (20ps/div).

In Fig. 18(left) the nonlinear characteristics of the first NOLM alone and of the two cascaded fibre loops are shown. The contribution of the second NOLM on the total characteristic is evident, making it very similar to the ideal step function. Moreover, in order to evaluate the advance in using two cascaded NOLMs, the all-optical schemes based on one or on two fibre loops can be compared in terms of BER using the set-up described in the following. Fig. 18(right) shows the BER curve measured at the input of the ODE, at the output of the first NOLM, and at the output of the second one. It can be noted that, in the considered case, the use of the additional ODE improves the performance of the conventional receiver of $1 \mathrm{~dB}$ and $1.5 \mathrm{~dB}$ at $\mathrm{BER}=10^{-9}$, using respectively one single NOLM and two cascaded NOLMs. The improvement in the signal quality passing through the two stages of the ODE is also evident from the eye-diagrams in the insets of Fig. 18.

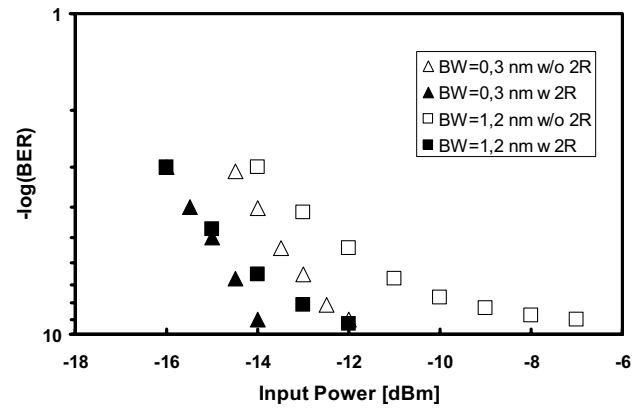

Fig. 19. BER measured with OSNR=15dB, for different signal bandwidths. 
To evaluate the benefits of using an additional ODE before a band-limited conventional receiver, a 10Gbps RZ system including a conventional 10GHz NRZ receiver can be used.

Fig. 19 and Fig. 20 show Bit Error Rate measurements obtained with the experimental set-up depicted in Fig. 17 using an RZ signal generated by a fibre mode-locked laser at 1551nm producing 4 ps-wide optical pulses at $10 \mathrm{GHz}$. The pulses have been then modulated with a $2^{31}-1$ pseudo-random bit sequence. This signal has been coupled with the noise emitted by an ASE source. Adjusting the noise level, different Optical Signal to Noise Ratios (OSNRs) have been realized. An optical filter then has been exploited to reshape the optical signal and to suppress out-of-band noise. Two different filters have been used, with a bandwidth of $0.3 \mathrm{~nm}$ and $1.2 \mathrm{~nm}$, generating final pulse widths of about $15 \mathrm{ps}$ and $5 \mathrm{ps}$ respectively. At the receiver input a variable optical attenuator has permitted to change the input power maintaining the OSNR unvaried.

The performance improvement due to the use of the NOLM based ODE can be evaluated by changing the signal bandwidth, in order to study the impact of different levels of mismatching between the bandwidths of the conventional receiver and the RZ signal. Fig. 19 reports the BER curves measured with and without the additional ODE, for 0.3nm and $1.2 \mathrm{~nm}$ pulse bandwidth, and a fixed OSNR=15dB (@ $0.1 \mathrm{~nm}$ resolution bandwidth). It is clear that the use of the ODE improves the performance of the receiver. Interpolations of the linear region of the BER curves allow to measure a negative power penalty at $B E R=10^{-9}$ of $1.5 \mathrm{~dB}$ and $2 \mathrm{~dB}$ for $0.3 \mathrm{~nm}$ and $1.2 \mathrm{~nm}$ signal bandwidth, respectively. This result confirms that the performance improvement due to the use of the additional ODE increases with the mismatching between signal and conventional receiver bandwidth. Moreover, in the case of $1.2 \mathrm{~nm}$ signal bandwidth, the conventional receiver reaches a floor in the BER curve, while using the proposed scheme this floor is not evident considering BER up to 10-10.

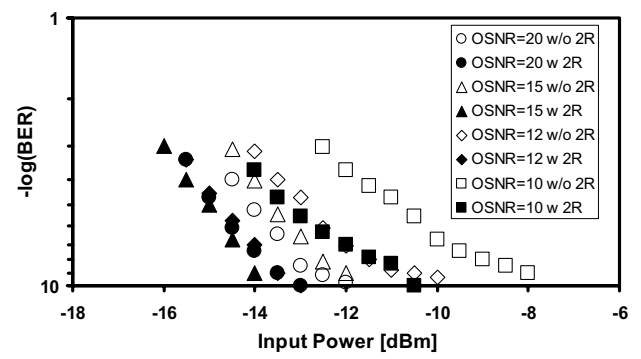

Fig. 20. BER measured with $0.3 \mathrm{~nm}$ signal bandwidth, for different OSNRs.

The behaviour of the proposed receiver can also be investigated for different OSNRs of the input signal. Experimental results are shown in Fig. 20 where the BER curves with and without the additional ODE are reported for OSNR=10, 12, 15 and $20 \mathrm{~dB}$, using $0.3 \mathrm{~nm}$ signal bandwidth. In each considered case the presence of the ODE improves the performance of the conventional receiver, with negative power penalties at $B E R=10^{-9}$ from $1.5 \mathrm{~dB}$ (OSNR $20 \mathrm{~dB}$ ) to $2 \mathrm{~dB}$ (OSNR 10dB), increasing for decreasing OSNR. It is important to notice that in the cases of OSNR=12, 15, and $20 \mathrm{~dB}$, the BER curves obtained using the additional ODE are compressed into $0.5 \mathrm{~dB}$. This is due to the characteristic of the scheme (Fig. 18), which can decide the signal correctly if the input noise is confined into its flat regions. As the noise 
exceeds these regions, the ODE can't decide correctly and the BER increases, as it is in the case of OSNR=10dB.

\subsection{R Regeneration}

The development of high bit rate optical transmission systems is facing strong limitations due to the impairments caused by fibre nonlinearities, chromatic and polarization mode dispersion. Therefore an in-line regeneration of the optical signal is necessary in order to increase the maximum transmission distance. For system bit rates higher than $40 \mathrm{Gbit} / \mathrm{s}$ the regeneration must be done in the optical domain, since the bandwidth of the electronic devices does not allow an optoelectronic conversion. Up to now, experimental results of alloptical regeneration have been presented for signal bit rates up to $40 \mathrm{Gbit} / \mathrm{s}$.

By splitting the regeneration process in three different steps based on the use of Nonlinear Optical Loop Mirrors, it is possible to perform the regeneration of a 160 Gbit/s OTDM signal.

An all-optical pulse regenerator is a functional block that receives a noisy and distorted signal and transmits an undistorted and noise-free signal. One of the possible methods to realize a signal regeneration is to transfer the information from the incoming data signal to a locally generated pulsed clock, whose characteristics in terms of pulse width, pulse shape and stability are suitable for the transmission. During this process the noise of the incoming data signal must be eliminated.

Because of the strong limitations due to low efficiency, long response time or high noise of the different regeneration techniques, it is very difficult to regenerate a $160 \mathrm{Gbit} / \mathrm{s}$ signal in a single step. Therefore a possible way to realize the regeneration at very high bit rate is to split the process in different steps. This can be done using a pedestal suppressor to eliminate the noise on the zero level, an inverting data transfer block to move the data to a synchronized clock, so that the noise on the one level is transferred to the zero level; and then another zero noise suppressor to eliminate this residual noise (Fig. 21). The advantage of this technique is that it requires well-known subsystems: two pedestal suppressors and a wavelength converter.

The functions of data transfer and zero noise suppression could both be realized exploiting nonlinear phenomena in semiconductor devices as the electro-absorption modulator or the semiconductor optical amplifier (Awad et al., 2000; Gavioli \& Bayvel, 2000). Due to their limited recovery time and to the low efficiency of their fast dynamics, it is preferable to use nonlinear effects in optical fibres.

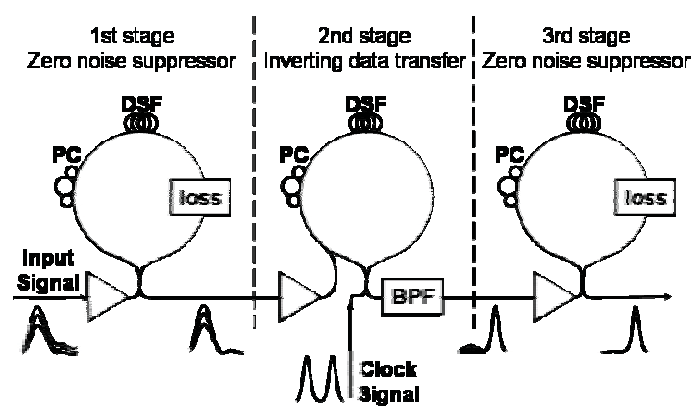

Fig. 21. Scheme of the working principle of the NOLM-based 3-stage regenerator. 
To realize the zero noise suppressors two NOLMs based on Self Phase Modulation in a Dispersion Shifted fibre can be used (Pelusi et al., 1999). The data transfer block is realized using a third NOLM exploiting Cross Phase Modulation in a DS fibre (Kaewplung \& Thipchatchawanwong, 2002).The input signal of the data transfer block is a pulsed clock, while the data stream is used as control signal inducing the phase shift. The data transfer block works in inverted logic thanks to an appropriate adjustment of the polarization of the two counterpropagating parts of the clock in the NOLM. The complete scheme of 3-stage NOLM-based regenerator is shown in Fig. 21.

Fig. 22 to Fig. 24 show experimental results obtained using two synchronized harmonic mode locked fibre lasers at $10 \mathrm{GHz}$, at $1557 \mathrm{~nm}$ and $1550 \mathrm{~nm}$ respectively. The signal pulses were modulated in a PRBS-driven Mach-Zehnder modulator and then distorted, so that the pulses to be regenerated were noisy and about 16 ps wide. The second pulsed laser was compressed with a higher order soliton compressor, and the pedestal was suppressed in a NOLM (Pelusi et al., 1999) obtaining a pulse width of 2 ps. Since it is not possible to visualize a $160 \mathrm{Gbit} / \mathrm{s}$ signal on the oscilloscope due to its limited bandwidth, the results shown are obtained at $10 \mathrm{Gbit} / \mathrm{s}$ using clock pulse width suitable for a $160 \mathrm{Gbit} / \mathrm{s}$ signal.

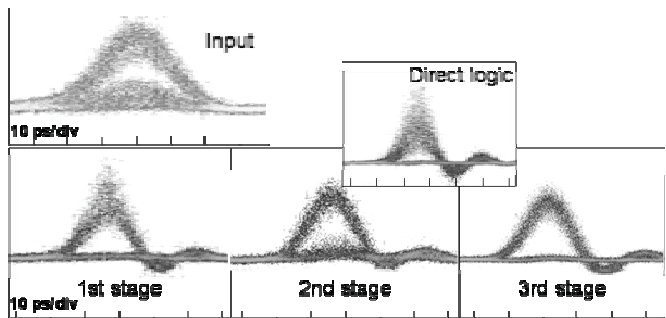

Fig. 22. Eye diagrams at the regenerator input and after each regenerator stage. Inset: 2 nd stage output eye diagram working in direct logic.

The improvement of the signal along the regenerator is shown in Fig. 22. In the first stage, the noise on the low level of the data signal is well suppressed using $1 \mathrm{~km}$ of dispersion shifted fibre and a mean input power of $20 \mathrm{dBm}$. In order to induce the same non linear effects, a $160 \mathrm{Gbit} / \mathrm{s}$ data signal should have a mean power lower than $27 \mathrm{dBm}$. The fibre length is a trade-off between high power requirements using a short fibre, and instability and pulse broadening using a long fibre. In the second stage the mean optical power of the data signal inducing the phase shift is $19.4 \mathrm{dBm}$. The DS fibre is about $1 \mathrm{~km}$ long since this fibre length, working with a $160 \mathrm{Gbit} / \mathrm{s}$ signal, is the best trade-off between cross-talk due to the walk-off, instability and pulse broadening on one hand, and high power requirements on the other. The data transfer block transfers the information to the clock signal with a logic inversion, so that the high level of the eye diagram is clean, while the zero level is still noisy. In the third stage, even this noise contribution is cancelled and the signal is regenerated, using $1 \mathrm{~km}$ of DS fibre and $15 \mathrm{dBm}$ mean power $(27 \mathrm{dBm}$ at $160 \mathrm{Gbit} / \mathrm{s})$. In the inset of Fig. 22 the output of the data transfer block working in direct logic is reported: in this case the input noise is transferred to the clock and amplified, and can not be reduced afterwards. The logic inversion instead moves the noise to the zero level allowing its reduction using the zero noise suppressor. 
Measuring the Q-factor of the eye diagrams, the bit error rate of the signal before and after the regeneration can be evaluated. In Fig. 23 the results in four different cases are reported, demonstrating the improvement in the quality of the signal. For each case, the input and output eye diagrams are reported in the insets. Best results show a BER improvement from $7 \cdot 10^{-4}$ to $9 \cdot 10^{-9}$. The performance of the regenerator depends on the input conditions: high noise on the zero level, on the one level or on both. In any case the regenerator ensured a BER improvement of a factor higher than $10^{3}$.

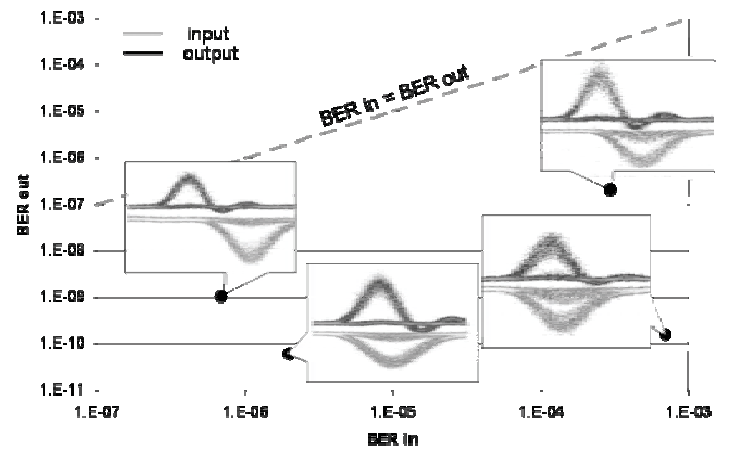

Fig. 23. BER improvement in four different cases. The insets show the eye diagrams for each considered case. Gray dots: regenerator input (inverting photodiode). Black dots: regenerator output.

To analyse the behaviour of the scheme in presence of cross-talk the signals can be multiplexed with 25 ps delay, as in a $40 \mathrm{Gbit} / \mathrm{s}$ system, so that the oscilloscope could still resolve the two pulses. Fig. 24 shows that the regenerator can transfer the information with a significant improvement of the eye diagram and a complete suppression of the cross-talk. A BER improvement of a factor $10^{6}$ is performed.

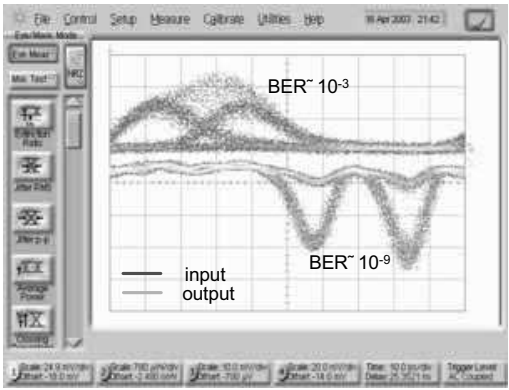

Fig. 24. Eye diagram and BER value for $40 \mathrm{Gbit} / \mathrm{s}$ transmitted pulses (black dots) and regenerated 2 ps pulses (gray dots). 


\section{References}

Agrawal, G. P. (1994). Nonlinear Fibre Optics, Accademic Press, 0-12-045142-5, San Diego, California, USA

Ahn, K. H.; Cao, X. D.; Liang, Y.; Barnett, B. C.; Chaikamnerd, S. \& Islam, M. N. (1997). Cascadability and functionality of all-optical low-birefringent nonlinear optical loop mirror: experimental demonstration. Journal of the Optical Society of America BOptical Physics, Vol. 14, No. 5, (May 1997) pp. 1228, 0740-3224.

Awad, E. S.; Cho, P. S.; Richardson, C.; Moulton, N.; Goldhar, J. (2000). Optical 3R regeneration with all-optical timing extraction and simultaneous wavelength conversion using a single electro-absorption modulator, Proceedings of ECOC2002, (2002), 6.3.2 Vol 3, 87-90974-65-4, Copenhagen, Denmark, 8-12 September 2002

Bogoni, A.; Scaffardi, M.; Ghelfi, P. \& L. Potì (2004). Nonlinear Optical Loop Mirrors: investigation solution and experimental validation for undesirable counterpropagating effects in all-optical signal processing. IEEE Selected Topics in Quantum Electronics, Vol. 10, No. 5, (Sept./Oct. 2004) pp. 1115-1123, 1077-260X

Bogoni, A.; Ghelfi, P.; Scaffardi, M. \& Potì, L. (2004). All-Optical Regeneration and Demultiplexing for $160 \mathrm{~Gb} / \mathrm{s}$ Transmission Systems Using a NOLM-Based ThreeStage Scheme. IEEE Selected Topics in Quantum Electronics, Vol. 10, No. 1, (Jan./Feb. 2004) pp. 192-196, 1077-260X

Chbat, M. W.; Hong, B.; Islam, M. N.; Soccolich, C. E. \& Prucnal, P. R. (1992). Ultrafast soliton-trapping AND gate. IEEE Journal of Lightwave Technology, Vol.10, No. 12, (Dec. 1992) pp. 2011-2016, 0733-8724

Collecutt, G.R. \& Drummond, P.D. (2000). Digital response in an all optical AND gate using parametric $\left(\chi^{(2)}\right)$ solitons, Proceedings of CLEO2000, pp. 194, 1-55752-634-6, San Francisco, California, USA 7-12 May 2000

Gavioli, G. \& Bayvel, P. (2002). Novel, high stability 3R all-optical regenerator based on polarization switching in a semiconductor optical amplifier Proceedings of ECOC2002, 7.3.2 Vol 3, 87-90974-65-4, Copenhagen, Denmark, 8-12 September 2002

Hall, K. L. \& Rauschenbach, K. A. (1998). 100-Gbitsbitwise logic. Optics Letters, Vol. 23, No. 16, (Aug. 1998) pp.1271-1273, 0146-9592

Ibrahim, T. A.; Grover, R.; Kuo, L. C.; Kanakaraju, S.; Calhoun, L. C. \& Ho, P. T. (2003). Alloptical AND/NAND logic gates using semiconductor microresonators, IEEE Photonics Technology Letters, Vol. 15, No. 10, (Oct. 2003) pp. 1422-1424, 1041-1135

Kaewplung, P. \& Thipchatchawanwong, P. (2002). Performance improvement of 40Gbit/s optical soliton transmission system by employing all-optically regenerative repeater using nonlinear optical loop mirror. Proceedings of APCCAS '02, pp. 215218 Vol. 2, 0-7803-7690-0, Denpasar, Bali, Indonesia, October 2002.

Meissner, M.; Rosch, M.; Schmauss, B. \& Leuchs, G. (2003). 12 dB of noise reduction by a NOLM-based 2-R regenerator, IEEE Photonics Technology Letters, Vol. 15, No. 9, (Sept. 2003) pp. 1297-1299, 1041-1135

Murakami, M.; Matsuda, T. \& Imai, T. (1998). Quarter terabit (25x10Gb/s) over 9288 km WDM transmission experiment using nonlinear supported RZ pulse in higher order fibre dispersion managed line, Proceedings of ECOC1998, pp. 77-81 Vol.3, 8489900-1 7-5, Madrid, Spain, 20-24 September 1998. 
Nayar, B. K.; Blow, K. J. \& Doran, N. J. (1991). All-optical switching in nonlinear fibre loop mirror devices. Optical Computing and Processing Vol. 1, No. 1, (Jan.-March 1991) pp. 81-89, 0954-2264

Nayar, B. K.; Finlayson, N. \& Doran, N. J. (1993). Concatenated All-optical Loop Mirror Switches. Journal of Modern Optics, Vol. 40, No. 12, (1993) pp. 2327-2332, 1362-3044

Olsson, B. E. \& Andrekson, P.A. (1998). Polarization-independent all-optical AND-gate using randomly birefringent fibre in a nonlinear optical loop mirror. Proceedings of OFC98, pp. 375-376, 1-55752-528-5, San Jose, California, USA 22-27 February 1998

Pattison, D.A.; Forysiak, W.; Kean, P. N.; Bennion, I. \& Doran, N. J. (1995). Soliton switching using cascaded nonlinear-optical loop mirrors. Optics Letters, Vol. 20, No. 1, (January 1995) pp.19-21, 0146-9592

Pelusi, M. D.; Matsui, Y. \& Suzuki, A. (1999). Pedestal suppression from compressed femtosecond pulses using a nonlinear fibre loop mirror, IEEE Journal of Quantum Electronics, Vol. 35, No. 6, (June 1999) pp. 867-874, 0018-9197

Rohde, H.; Lehmann, C. \& Schairer, W. (2004). Towards a meshed ultra high speed TDM optical network: concept, OADM architecture and proof of principle, Proceedings OFC2004, TuH6, 1-55752-772-5,Los Angeles, California, USA, 23-25 February 2004.

Sakamoto, T.; Futami, F.; Kikuchi, K.; Takeda, S.; Sugaya, Y. \& Watanabe, S. (2001). Alloptical wavelength conversion of 500 -fs pulse trains by using a nonlinear-optical loop mirror composed of a highly nonlinear DSF. IEEE Photonics Technology Letters, Vol. 13, No. 5, (May 2001) pp. 502-504, 1041-1135

Scaffardi, M.; Ghelfi, P.; Bogoni, A. \& Potì, L. (2004). Investigation and solution for undesirable counter-propagating effects in nonlinear optical loop mirrors. Proceedings of CLEO 2004, Vol. 2, pp. 2-3, 1-55752-777-6, San Francisco, California, USA 17-19 May 2004

Stubkjaer, K. E. (2000). Semiconductor optical amplifier-based all-optical gates for highspeed optical processing. IEEE Selected Topics in Quantum Electronics, Vol. 6, No. 6, (Nov./Dec. 2000) pp. 1428-1435, 1077-260X

Webb, R. P.; Manning, R. J.; Maxwell, G. D. \& Poustie, A. J. (2003). 40 Gbit/s all-optical XOR gate based on hybrid-integrated Mach-Zehnder interferometer. IEE Electronics Letters, Vol. 39, No, 1, (Jan. 2003) pp.79-81, 0013-5194

Winzer, P. J.; Pfennigbauer, M.; Strasser, M. M. \& Leeb, W.R. (2001). Optimum filter bandwidths for optically preamplified NRZ receivers, IEEE Journal of Lightwave Technology., Vol. 19, No. 9, (Sept. 2001) pp. 1263-1273, 0733-8724 


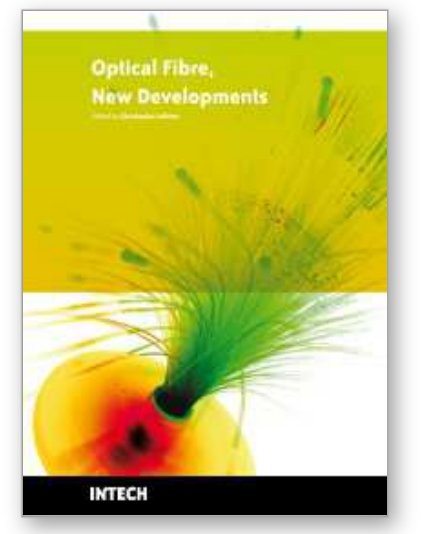

\section{Optical Fiber New Developments}

Edited by Christophe Lethien

ISBN 978-953-7619-50-3

Hard cover, 586 pages

Publisher InTech

Published online 01, December, 2009

Published in print edition December, 2009

The optical fibre technology is one of the hop topics developed in the beginning of the 21 th century and could substantially benefit applications dealing with lighting, sensing and communication systems. Many improvements have been made in the past years to reduce the fibre attenuation and to improve the fibre performance. Nowadays, new applications have been developed over the scientific community and this book fits this paradigm. It summarizes the current status of know-how in optical fibre applications and represents a further source of information dealing with two main topics: the development of fibre optics sensors, and the application of optical fibre for telecommunication systems.

\section{How to reference}

In order to correctly reference this scholarly work, feel free to copy and paste the following:

Antonella Bogoni, Francesco Fresi, Paolo Ghelfi, Emma Lazzeri, Luca Poti and Mirco Scaffardi (2009). Fibre Based Schemes for Ultrafast Subsystems: Nonlinear Optical Loop Mirrors Traditional Design and Novel Applications, Optical Fiber New Developments, Christophe Lethien (Ed.), ISBN: 978-953-7619-50-3, InTech, Available from: http://www.intechopen.com/books/optical-fiber-new-developments/fibre-based-schemes-forultrafast-subsystems-nonlinear-optical-loop-mirrors-traditional-design-and-n

\section{INTECH}

open science | open minds

\section{InTech Europe}

University Campus STeP Ri

Slavka Krautzeka 83/A

51000 Rijeka, Croatia

Phone: +385 (51) 770447

Fax: +385 (51) 686166

www.intechopen.com

\section{InTech China}

Unit 405, Office Block, Hotel Equatorial Shanghai

No.65, Yan An Road (West), Shanghai, 200040, China 中国上海市延安西路65号上海国际贵都大饭店办公楼 405 单元 Phone: +86-21-62489820

Fax: +86-21-62489821 
(C) 2009 The Author(s). Licensee IntechOpen. This chapter is distributed under the terms of the Creative Commons Attribution-NonCommercial-ShareAlike-3.0 License, which permits use, distribution and reproduction for non-commercial purposes, provided the original is properly cited and derivative works building on this content are distributed under the same license. 